\title{
The Effects of Guilt and Sadness on Sugar Consumption
}

\author{
Sarah Lefebvre \\ slefebvre@murraystate.edu \\ Jonathan Hasford \\ University of Tennessee, Knoxville \\ Ze Wang \\ University of Central Florida, ze.wang@ucf.edu
}

Follow this and additional works at: https://digitalcommons.murraystate.edu/faculty

\section{Recommended Citation}

Lefebvre, S., Hasford, J., \& Wang, Z. (2019). The effects of guilt and sadness on sugar consumption. Journal of Business Research, 100, 130-138.

This Journal Article is brought to you for free and open access by the Faculty Works at Murray State's Digital Commons. It has been accepted for inclusion in Faculty \& Staff Research and Creative Activity by an authorized administrator of Murray State's Digital Commons. For more information, please contact msu.digitalcommons@murraystate.edu. 


\begin{abstract}
This research examines how the discrete negative emotions of guilt and sadness impact individual preference for carbohydrates, specifically in the form of sugar. Using Cognitive Appraisal Theory and research in biological psychology, we identify how these two discrete emotions influence the release of cortisol, which impacts sugar preferences. The results of four studies indicate that consumers select and prepare foods with higher amounts of sugar when experiencing sadness relative to when they feel guilt. Implications for public policy as well as marketing practitioners are discussed.
\end{abstract}

Keywords: Sadness; Guilt; Food Preference; Sugar Consumption

Word Count: 6,364 


\section{The Effects of Guilt and Sadness on Sugar Consumption.}

\section{Introduction}

Negative affective states lead individuals to engage in a variety of coping behaviors. For instance, anger results in impulsivity (Macht, 1999), loneliness is linked to valuing possessions

(Pieters, 2013), and experiencing fear leads to stronger brand attachment (Dunn \& Hoegg, 2014). Within the food domain, extensive evidence has documented how individuals consume unhealthy but hedonically rewarding foods as a coping strategy to alleviate their negative feelings (Bublitz, Peracchio, \& Block, 2010; Mantau, Hattula, \& Bornemann, 2018; see Cardi, Leppanen, \& Treasure, 2015 for a meta-analysis review). For example, feelings of guilt towards indulgent foods can reduce an individual's perceptions of control over their eating habits and ultimately lead them to gain weight over an 18-month period (Kuijer \& Boyce, 2014). Relatedly, feelings of sadness can lead individuals to consume greater amounts of popcorn and candy while watching a movie (Garg, Wansink, \& Inman, 2007) and more readily dine at trendy restaurants perceived to be indulgent (Salerno, Laran, \& Janiszewski, 2014). Unfortunately, the consequences of these food consumption behaviors can have detrimental impacts on consumer health. The Center for Disease Control and Prevention (CDC) estimates that more than 93 million Americans are obese (CDC, 2017b), which places nearly 40\% of the adult US population at an increased risk of heart disease and other major health issues (CDC, 2016).

Not all food consumption is equally concerning though. Research shows that consumption of high fat and high sugar snacks are a factor in the staggering number of overweight and obese individuals (Reichenberger et al., 2018). Further, studies of nutritional 
health estimate that 100 million Americans had diabetes or prediabetes (CDC, 2017a), of which sugar consumption is a primary contributor (Bunim, 2013). While the American Heart Association (AHA) recommends sugar consumption should be no more than 6 teaspoons per day for women and 9 teaspoons per day for men, actual sugar consumption is approximately 20 teaspoons per day for an average adult in the US (AHA, 2018). Furthermore, carbohydrates in the form of sugar do not induce satiety as compared to fat or carbohydrates in the form of fiber (Van Dam \& Seidell, 2007). Thus, individuals may consume sugar to alleviate their negative affective states, only to experience ongoing negative affect and consequently engage in continued indulgence. Evident from these observations, there is a pressing need to examine how consumers' negative emotions impact their consumption of different macronutrients, especially refined carbohydrates in the form of sugar.

The current research extends the discrete emotion literature by examining how two specific negative emotions (guilt and sadness) impact individual preferences for consumption of carbohydrates, particularly in the form of sugar. Using Cognitive Appraisal Theory (Lazarus, 1991; Smith \& Ellsworth, 1985) and findings from biological psychology, this research examines the effects of guilt and sadness simultaneously, explores the underlying mechanisms, and measures their impact in both food preferences and food preparation involving sugar. The results of four studies indicate that consumers select and prepare foods with higher amounts of sugar when experiencing sadness relative to when they feel guilt. Together, our findings extend the literature on discrete negative emotions in food consumption, which has generally found that negative affective states elicit a desire to consume hedonic foods regardless of their macronutrient content (e.g. Cardi, Leppanen, \& Treasure, 2015; Cools, Schotte, \& McNally, 1992; Lowe \& Fisher Jr., 1983; Salerno, Laran, \& Janiszewski, 2014; Tice, Bratslavsky, \& 
Baumeister, 2001). Further, this research contributes to the literature on discrete emotions by identifying how both emotion appraisals and physiological changes jointly influence behavior. Prior research related to guilt and/or sadness within the food domain has primarily focused on psychological explanations of how these discrete emotions impact indulgence. Thus, our findings contribute to a more complete understanding of the mental and physical changes that occur when guilt and sadness become salient.

Below, we begin with an overview of the extant research on guilt and sadness. We then discuss the physiological responses to guilt and sadness and propose the corresponding impact on sugar consumption. We test our hypotheses in four empirical studies. To conclude, the theoretical and practical implications of the findings are discussed.

\section{Conceptual Background and Hypotheses Development}

\subsection{Guilt and Sadness}

Guilt and sadness represent two discrete negative emotions that differentially impact individual behavior. Guilt is classified as a self-conscious emotion, whereby individuals engage in self-evaluation in response to their negative feeling state (Hung \& Mukhopadhyay, 2012; Tangney \& Dearing, 2003). Guilt often leads an individual to attribute their negative affective state to themselves. Research has shown individuals experiencing guilt felt they had violated a moral standard and felt a sense of responsibility for the source of the emotion (Tangney, Miller, Flicker, \& Barlow, 1996). Further, there is a perceived lack of control with guilt that may result in risk-taking behaviors to reestablish self-control (Kouchaki, Oveis, \& Gino, 2014). Guilt has also been found to activate a desire to improve the self, resulting in a preference for self- 
improvement products such as fitness apps, functional beverages and sleep promoting tablets (Allard \& White, 2015). The involvement in the resulting negative affective state, leads to behavior-specific appraisals associated with their feeling state (Han, Duhachek, \& Agrawal, 2014). As a result, individuals may seek to punish or deprive themselves in response to feeling guilty (Carveth, 2001). For instance, when guilt was the result of one's own action, local (vs. global) appraisals were found to influence consumer preference for feasible attributes (e.g., an affordable price) over desirable attributes (e.g., a product you really want) when selecting a product (Han, Duhachek, \& Agrawal, 2014).

However, food consumption research has shown that guilt does not always lead to adaptive or motivational behavior (Kuijer \& Boyce, 2014). For example, individuals may form cognitive associations between guilt and the pleasure experienced from hedonic consumption which increases the consumption of indulgences such as chocolate candy and truffles (Goldsmith, Cho, \& Dhar, 2012). Due to these positive associations, feeling guilty may be counterproductive in the long-run to an individual's dietary goals (Kuijer, Boyce, \& Marshall, 2015). Specifically, individuals who associated chocolate cake with guilt compared to those who associated chocolate cake with celebration had weaker intentions to eat healthy, reported less behavioral control, and were less successful at maintaining their weight in the long term (Kuijer \& Boyce, 2014). Collectively, results of guilt research within the food domain show that feelings of guilt associations with various hedonic foods may ultimately increase the indulgence of those items.

In contrast to self-attribution, sadness entails more automatic responses related to a negative, low arousal feeling toward one's current situation (Goldberg, Lerner, \& Tetlock, 1999). Unlike guilt, sadness is associated with a core theme of loss and helplessness that can extend 
beyond single situations and appraisals to influence decision making (Garg \& Lerner, 2013).

Sadness can perpetuate behaviors that are detrimental to oneself (Leith \& Baumeister, 1996) and bias decision making toward immediate gratification and reward-centered behaviors (Lerner, Li, \& Weber, 2012; Zemack-Rugar, Bettman \& Fitzsimons, 2007). For instance, research has shown sadness reduces patience (Lerner, Li, \& Weber, 2012) and increases preferences for entertainment products (e.g. CDs/DVDs; Zemack-Rugar, Bettman, \& Fitzsimons, 2007). Selfregulation theory suggests sadness leads to overconsumption due to activation of mood regulation in the form of indulgent consumption (Baumeister, Heatherton, \& Tice 1994).

In the food domain, sadness (compared to happiness or neutral affective states) can lead to increased consumption of hedonic foods. For instance, research has shown sadness increases the desire for consumption of indulgent foods despite their caloric density or perceived flavor when hunger levels are controlled for (Ershadi, Russell, \& Herz, 2017). A variety of reasons have been attributed to the impact of sadness on hedonic consumption. Sadness can enhance consumption of indulgences such as buttered popcorn and M\&Ms because of the associated mood-changing cues (Garg, Wansink, \& Inman, 2007; Salerno, Laran, \& Janiszewski, 2014). Other research identifies that self-reported helplessness mediated the effect of sadness on increased candy consumption (e.g., chocolate), as individuals consume hedonic foods as a form of compensatory consumption (Garg \& Lerner, 2013).

Thus, like guilt, research has shown sadness increases indulgence of various hedonic foods. However, these studies do not examine the role of food product attributes, such as whether sugar is the source of indulgence or whether differences in guilt or sadness impact preferences for specific macronutrients. To provide insights on these issues, we use Cognitive Appraisal 
Theory (Smith \& Ellsworth, 1985) to explore how these discrete emotions influence an individual's physiological responses to sugar.

\subsection{Cognitive Appraisal Theory and Stressors}

Cognitive Appraisal Theory proposes that emotions are evoked based on evaluations of the events leading to the emotion (Lazarus, 1991; Smith \& Ellsworth, 1985). Four factors are considered in the appraisal process, including if the outcome of the situation is good or bad (outcome desirability), if oneself or someone else is responsible for the outcome (i.e. outcome agency), the certainty of the event, and perceptions of the event's fairness (Watson \& Spence, 2007). Further, individuals will appraise the demands, followed by an assessment of the resources they can allocate to the situation (Harvey, Nathens, Bandiera, \& LeBlack, 2010). These appraisals subsequently determine an individual's response to the experienced emotion. For example, individuals often experience stress when negative emotions are perceived to be relevant to one's well-being (Lazarus \& Folkman, 1984).

Stress in general has been linked to both over- and under consumption of foods (Reichenberger et al., 2018). Stressors elicited from negative emotions can impact behavior depending how they are categorized by individuals. Specifically, stressors can be categorized as either challenge stressors or threat stressors (Adam \& Epel, 2007; Gaab, Rohleder, Nater, \& Ehlert, 2005; Harvey et al., 2010). Developed from Cognitive Appraisal Theory, the Biopsychosocial Model of Challenge and Threat links cognitive appraisals with physiological responses based on situational evaluations of perceived demands and available resources (Blascovich, 2013). When an individual appraises resources to be greater than demands for a situation, the result is challenge stress (Harvey et al., 2010). Challenge stressors are viewed as 
demanding (Adam \& Epel, 2007), but individuals believe they can cope with and have control over these stressors (Blascovich \& Mendes, 2000). Further, challenge stressors occur when the situation influences self-worth (Blascovich et al., 2001). We categorize guilt as a challenge stressor, given that guilt is a self-conscious emotion that influences perceptions of control and self-worth (Carveth, 2001; Han, Duhachek, \& Agrawal, 2014; Kouchaki, Oveis, \& Gino, 2014).

Threat stressors, in contrast, are seen as uncontrollable, associated with distress, feeling defeated (Adam \& Epel, 2007) and maladaptive tendencies (Blascovich \& Mendes, 2000). In the case of threat stressors, appraisal of the situation has led the individual to realize demands outweigh resources and there is a significant potential for loss (Harvey et al., 2010). Given that individuals perceive a lack of control when experiencing sadness (Garg \& Lerner, 2013), and potential loss becomes a primary concern (Salerno, Laran, \& Janiszewski, 2010), we categorize sadness as a threat stressor. To understand how these different stressors influence sugar consumption, next we examine how these stressors are linked to distinct physiological responses.

\subsection{Physiological Response to Guilt and Sadness}

Prior research has linked both challenge and threat stressors to physiological responses. For example, when individuals detect a challenge stressor, the sympathetic-adrenomedullary (SAM) system is activated within the brain (Adam \& Epel, 2007; Blascovich \& Tomaka, 1996). The activation of the SAM system leads to the release of adrenaline, which decreases digestion within the body as the focus is shifted to the muscles and blood flow (Adam \& Epel, 2007). Several studies have noted that adrenaline suppresses an individual's appetite and reduces food consumption (Russek, Lora-Vilchis, \& Isla-Chaires, 1980; Russek, Mogenson, \& Stevenson, 1967; Russek, Stevenson, \& Mogenson, 1968). 
Conversely, stress perceived as threating has been found to increase consumption. Egothreating stress has been linked with increased snacking of high fat, high sugar foods (O'Connor et al., 2008). Threat stressors activate the hypothalamus pituitary adrenal (HPA) axis, which inhibits the release of adrenaline and leads to the release of cortisol (Blascovich \& Tomaka, 1996; Dallman, 2010; Harvey et al., 2010). For example, this physiological-emotion response of threat stress and cortisol has been shown in research with students (Dickerson, Mycek, \& Zaldivar, 2008) and medical professionals (Harvey et al., 2010), where induced threat stress was associated with an increase in cortisol levels. Cortisol secretion has been found to increase caloric intake by increasing motivation to consume highly palatable foods, such as those high in fat and sugar content (Epel, Lapidus, McEwen, \& Brownell, 2001; Tempel \& Leibowitz, 1994). Specifically, cortisol has been found to enhance preferences for sweet flavors (Epel et al., 2001), which typically contain high levels of sugar.

Together, the combination of discrete emotion appraisals and physiological responses to various forms of negative affect lead us to our core predictions. Since sadness is appraised to be a threat stressor, this should lead to the release of cortisol. This physiological response should lead individuals to prefer higher levels of sugar in response to the threat stressor. In contrast, experiencing guilt, a challenge stressor, will not elicit a preference for high sugar foods. Stated formally:

H1: When experiencing sadness (vs. guilt) individuals will experience a preference for foods with a high sugar content.

H2: The influence of sadness (vs. guilt) on preference for high sugar foods will be mediated by the physiological responses to emotional appraisals.

To test our predictions, four studies were conducted. In study 1, we investigated how sadness compared to guilt and a control influences choice of a high- (vs. low-) sugar snack. In 
study 2 , we replicated our findings of study 1 and present process evidence of our proposed physiological mechanisms. Study 3, demonstrates that the effect extends to both hedonic and non-hedonic foods. Then, in study 4 we generalize our findings to examine how guilt and sadness impact sugar usage in the preparation of food. The studies are described next.

\section{Study 1}

Study 1 was designed to provide initial evidence that discrete negative emotions (guilt vs. sadness) are differentially associated with sugar consumption. Two-hundred and fifty undergraduate students were randomly assigned to a single-factor (emotion manipulation: guilt/sadness/control) between-subjects design. Study 1 was included at the end of a series of lab sessions where undergraduates participated for extra credit.

\subsection{Method}

Participants were initially instructed to complete a writing task. The written recall task was used to manipulate felt emotions and was based on prior research (Roseman, Wiest, \& Swartz, 1994). In the emotion primed conditions, participants were asked to "Please recall a time in your personal life where you behaved in a way that made you feel [guilty/sad]. Please describe the details about this situation that made you feel [guilty/sad].” In the control condition, participants were asked to "Please recall what you did last weekend." All participants were also instructed to "Please provide as many details as possible so that a person reading your entry would understand the situation and how you felt" to further strengthen our emotion manipulation. 
Prior to collecting study 1, we conducted a pretest of the written recall using Amazon Mechanical Turk (MTurk). Forty participants were recruited to participate in exchange for monetary compensation. Participants were randomly assigned to complete either the guilt or sadness writing manipulation and then indicate how sad and guilty they felt on a five-point scale $(1=$ not at all, $5=$ extremely). T-tests revealed participants in the guilt condition reported feeling significantly more guilt $(M=3.27)$ relative to participants in the sadness condition $(M=2.21$; $t(39)=2.41, p<.05)$, while participants in the sadness condition reported significantly more sadness $(M=3.16)$ relative to the guilt condition $(M=2.32 ; t(39)=2.11, p<.05)$, after completing the recall task. Thus, our writing task was effective at manipulating the desired emotions.

Following the emotion manipulations in our main study, participants were offered a snack for participating in the lab session. Participants were given four snack choices, including two low sugar snacks (regular potato chips and mini pretzels, both with <1 gram of sugar per 30 gram serving), and two high sugar snacks (M\&Ms [19 grams per 30 gram serving] and mini chocolate chip cookies [11 grams per 30 gram serving]). All snack options were hedonic in nature to specifically examine the impact of discrete negative emotions on sugar consumption. Participants made their selection in the study questionnaire and reported their choice to the researcher who provided them with their chosen snack.

\subsection{Results}

Four participants were removed for not following instructions to complete the written recall task, leaving 246 usable responses in the analysis. To examine the relationship of discrete emotions on snack choice, a logistic regression was conducted with emotion condition predicting 
snack choice $(0=$ low sugar snack, $1=$ high sugar snack). Results revealed a significant effect of emotion condition on choice $($ Wald $=6.02, p<.05)$, where those in the sad condition chose the high sugar items more frequently than participants in the guilt condition $\left(\mathrm{P}_{\text {sad }}=74 \%\right.$ vs. $\mathrm{P}_{\text {guilt }}=$ $60 \%$, Wald $=3.57, p=.059)$ and the control condition $\left(\mathrm{P}_{\text {control }}=56 \%\right.$, Wald $\left.=5.56, p<.05\right)$. Choice in the guilt and control conditions did not significantly differ from each other (Wald= $.211, p=.65)$, suggesting when experiencing guilt preference for sugar does not differ from a neutral emotional state. Together, these results support $\mathrm{H} 1$.

\subsection{Discussion}

Study 1 provides initial evidence that guilt and sadness differentially impact preference for sugar consumption. Specifically, participants primed with sadness were more likely to choose a snack option that was high in sugar than those primed with guilt or those in a control condition. These results suggest, while both guilt and sadness are negatively valenced discrete emotions, they influence preference for sugar in distinct ways. In study 2, we generalize this effect and initially examine our conceptual model.

\section{Study 2}

Study 2 was designed to generalize the results of study 1 and provide evidence for why sadness enhanced sugar consumption compared to guilt. Specifically, we were interested in whether our observed effects were mediated by participants' desire for sugar as a physiological response to the discrete emotion of sadness. Two-hundred and thirteen undergraduates were 
randomly assigned to a single-factor (emotion manipulation: guilt/sadness) between-subjects design.

\subsection{Method}

In study 2, we generalized our manipulation of discrete emotions by using a sentence unscrambling task based on previous research (Briley \& Aaker, 2006; Srull \& Wyer, 1979). Participants were given 10 sentences with the words out of order (e.g. "friends/felt guilty/Matt/for/deceiving/his") and asked to unscramble each sentence. In each condition, five of the sentences were associated with either guilt or sadness to prime the respective emotion. The sentences are available in the Appendix.

Following the sentence unscrambling task, participants were asked to indicate their preference for three high sugar snacks (cookies, brownies, and Swedish fish) on 7-point scales (1 $=$ not at all, $7=\mathrm{a}$ lot $)$. Preferences for the high sugar snacks served as our primary dependent measure of sugar preference.

Then, we examined whether physiological responses underlie our relationship between sadness and sugar consumption. As prior research has linked physiological responses to cravings for various types of food (e.g., Nederkoorn, Smulders, \& Jansen, 2000; Yanovski, 2003), we measured sugar cravings in participants given its link to cortisol secretion (Epel et al., 2001). Thus, participants completed a 3-item measure of sugar cravings. The items $(\alpha=.89)$ are available in the Appendix.

\subsection{Results}


4.2.1 Preference for high sugar snacks. To examine preferences for each snack, a repeated-measures ANOVA was conducted. Results revealed a significant main effect of emotion condition on snack preferences $(F(1,211)=6.70, p=.01)$. Post-hoc comparisons revealed the average snack preference for participants in the sadness condition $(M=4.04)$ was significantly higher relative to participants in the guilt condition $(\mathrm{M}=3.52, p=.01)$. This finding further supports $\mathrm{H} 1$.

4.2.2 Mediation analysis. To assess the mediating effect of sugar cravings, we used PROCESS Model 4 (Hayes, 2013) with 5000 bootstrap samples. Results (all coefficients are unstandardized) are available in figure 1 . The main effect of emotion condition $(1=$ guilt, $2=$ sadness) on sugar cravings was significant $(\mathrm{a}=.45, p=.048)$. Sugar cravings were positively related to the average preference for the high-sugar snacks $(b=.49, p<.001)$. Furthermore, while the indirect effect of emotion condition on preference for high sugar snacks was significant $($ effect $=.22,95 \% \mathrm{CI} .009$ to .444$)$, the direct effect of emotion condition on snack preference became nonsignficant (effect $=.303, p=.077$ ). Together, these findings provide evidence of full mediation, in support of $\mathrm{H} 2$ and our proposed conceptual model.

\section{[Insert Figure 1 About Here]}

4.2.3 Post-hoc discriminant analysis. To ensure that sugar cravings and snack preferences were distinct constructs, we also conducted a discriminant analysis. In our analysis, we compared two models: the first model included all six items measuring sugar cravings and snack preferences as a single construct and a second model with sugar cravings and snack preferences as separate constructs. Results revealed significantly improved model fit for the 
second model $\left(\Delta \chi^{2}=41.99, p<.001\right)$, suggesting that sugar cravings and snack preferences are distinct constructs.

\subsection{Discussion}

Study 2 provides additional support for our core predictions. Using a distinct manipulation of guilt and sadness from study 1, we replicated our finding that individuals who are experiencing sadness (vs. guilt) are more likely to prefer a high-sugar snack. Additionally, we provided process evidence in support of our proposed model. Given that activation of cortisol is linked to sugar cravings (Epel et al., 2001), we use sugar cravings as a proxy for our proposed physiological process and show that sugar cravings mediate the relationship between sadness and sugar consumption. Next, we further generalize these findings by examining how the impact of sadness on sugar preference extends to non-hedonic food products.

\section{Study 3}

Study 3 was designed to further support our conceptual model by manipulating the same food as either a high-sugar or a low-sugar food. In addition, the food is non-hedonic in nature to demonstrate that the effect is not limited to indulgent, hedonic foods. We predict that when a food item is presented as a high-sugar item, individuals experiencing sadness will be more favorable towards the item than those who are experiencing guilt. However, when the same food is presented as a low-sugar item, the effect will be attenuated. To test these predictions, 217 undergraduates were randomly assigned to a 2 (emotion manipulation: guilt/sadness) x 2 (food: high sugar/low sugar) between-subjects design. 


\subsection{Method}

The emotion manipulation was the same sentence unscrambling task as in study 2 . Following the sentence unscrambling task, participants viewed a nutrition label for Greek yogurt. In the high sugar condition, the yogurt had 25 grams of sugar and 5 grams of protein in a single serving. In the low sugar condition, the yogurt had 5 grams of sugar and 25 grams of protein in the same serving. Calories (and fat content) were thus held constant across conditions at 160 calories. The labels are available in the Appendix. After viewing the label, participants were asked "if you had this yogurt right now, how much would you want to consume" on a 7-point scale $(1=$ none at all, $7=\mathrm{a}$ lot $)$. To conclude, participants were asked to rate the sugar content of the yogurt on a 7-point scale $(1=$ very low, 7 = very high $)$.

\subsection{Results}

5.2.1 Manipulation check. To begin, we examined whether our nutrition label manipulation had its intended effect. An ANOVA with the emotion and food condition variables predicting sugar perceptions was conducted. Results revealed a main effect of food condition $(F(1,213)=39.33, p<.001)$, as participants in the high sugar condition rated the food as having higher sugar content $(\mathrm{M}=4.31)$ relative to participants in the low sugar condition $(\mathrm{M}=2.94)$. Furthermore, the main effect of emotion condition and the interaction of emotion and food were nonsignificant $(p s>.24)$.

5.2.1 Food preferences. Next, we examined how consumption preferences were impacted by guilt and sadness. An ANOVA with the emotion and food condition variables predicting consumption preferences was conducted. Results (see figure 2) revealed a significant 
interaction of emotion and food $(F(1,213)=8.42, p<.01)$. Contrast analyses revealed that for participants in the high sugar condition, individuals experiencing sadness wanted to consume more yogurt $(M=3.97)$ relative to participants experiencing guilt $(M=3.21, p=.05)$. This result further supports H1. Additionally for participants in the low sugar condition, individuals experiencing guilt wanted to consume more yogurt $(\mathrm{M}=3.54)$ relative to participants experiencing sadness $(\mathrm{M}=2.74, p<.05)$.

[Insert Figure 2 About Here]

\subsection{Discussion}

Study 3 provides additional support for our predictions regarding guilt, sadness, and sugar preferences using a non-hedonic food product that varied in sugar content. The results demonstrate a greater preference for high-sugar items when experiencing sadness compared to guilt. Interestingly, preference for low-sugar items was enhanced when participants experienced guilt. Since challenge stressors, like guilt, are related to behaviors that are controllable by the individual, this reversal in effect may be due to the individual's attempt to gain control through their consumption behaviors and correct the guilt being experienced. Further, sugar is quickly digested and absorbed by the body after consumption, while decreased appetite is usually experienced as a consequence to adrenaline released in response to a challenge stressor such as guilt. Thus, a second possible explanation could entail that the preference for low sugar is in response to the decreased appetite and desire to avoid quick digestion and absorption. However, this guilt effect is not central to our conceptual framework and further discussion is thus omitted. Next, we test how feelings of sadness can impact sugar use in the preparation of foods. 


\section{Study 4}

While studies 1 - 3 show that individuals have a greater preference for high-sugar snacks when experiencing sadness (vs. guilt), an alternative explanation exists regarding our findings. Individuals could have made their choices on the basis of something other than sugar, such as the perceived tastiness of the foods. To rule out this alternative explanation, study 4 was designed to investigate how our observed effects extend to the preparation of food and whether participants explicitly add more sugar to a meal when feeling sad. Sixty-six undergraduates were randomly assigned to a single-factor (emotion manipulation: guilt/sadness) between-subjects design.

\subsection{Method}

Upon entering the lab, participants were seated at an individual workstation and provided with a paper instructions packet, which randomly assigned them to one of two emotion conditions. Participants then completed the written recall task used in study 1. After completing the emotion manipulation, participants were provided with a scenario of a company looking to open a "create-your-own-shake bar" similar to popular self-serve frozen yogurt bars (see Appendix). Participants entered a private room with four labeled clear cylinders on a table with a clear one tablespoon scoop with each item; (1) protein powder, (2) sugar, (3) bone builder: with calcium and (4) immune booster: with vitamin C. Participants were directed to use the provided cup and scoop as much or as little of each item they wished to include in their shake. They were also able to select additional items and a flavor on the provided instruction sheet. While each participant scooped from the cylinders, a proctor was monitoring and observing each 
participant's selections through a one-way mirror and recording the number of scoops selected for each item. The stimuli table was set up approximately two feet away from the one-way mirror providing the proctor with a detailed view of the participants' movements and selections. Further, the provided scoop was clear allowing the proctor to view how much the participant had selected in each scoop. Scoops were recorded to the nearest quarter (e.g., .25 scoop, .5 scoop). One scoop was equivalent to one tablespoon, where one tablespoon of sugar consists of 12.5 grams. The amount of sugar participants added to their shake served as the focal dependent variable. After selecting the ingredients, participants completed the paper form with additional items they wanted in the shake and were then dismissed from the lab session.

\subsection{Results}

To compare the amount of sugar scooped into each shake by condition, a t-test was conducted. Results revealed that participants in the sadness condition ( $M=4.62$ grams $)$ added significantly more sugar to their shake relative to participants in the guilt condition $(\mathrm{M}=2.08$ grams, $t(64)=2.02, p=.047)$. This finding further supports $\mathrm{H} 1$.

\subsection{Discussion}

Study 4 provides additional evidence that sadness, relative to guilt, influences sugar consumption. Using a "create-your-own-shake" bar, participants who were primed with sadness added more sugar to their shakes than participants primed with guilt. This finding provides additional support for our conceptual model of discrete emotions and sugar consumption.

\section{General Discussion}


Discrete emotions such as guilt and sadness can lead individuals to engage in coping behaviors, such as the consumption of unhealthy yet hedonically rewarding foods (Aydinoglu \& Krishna, 2011; Cornil \& Chandon, 2013; Garg \& Lerner, 2013; King, Herman, \& Polivy, 1987; Troisi \& Gabriel, 2011). The current research extends these findings by highlighting how guilt and sadness differentially impact preferences for specific macronutrients beyond merely indulgent foods. Four studies demonstrate that individuals who feel sad (vs. guilty) demonstrate a greater preference for sugar both in the choice and preparation of food. Together, these findings provide important theoretical and practical implications.

\subsection{Research Implications}

Our results extend research on discrete emotions by identifying how both emotion appraisals and physiological changes jointly influence behavior. Specifically, sadness elicits an appraisal consistent with that of a threat stressor (Dallman, 2010; Harvey et al., 2010), which activates cortisol and a subsequent desire for palatable foods, specifically those high in sugar (Epel et al., 2001). However, because guilt elicits an appraisal consistent with that of a challenge stressor (Adam \& Epel, 2007), adrenaline is produced by the body which curbs one's appetite for high sugar foods. Together, these appraisals and physiological responses combine to distinctively impact one's consumption of sugar. Specifically, preference for sugar increases when experiencing sadness compared to when experiencing guilt and a neutral control. We find support for this effect with both hedonic (e.g., chips, candy) and non-hedonic (e.g., yogurt) food products. In doing so, our research extends prior findings that suggest guilt primes, relative to sadness primes, can impact indulgence (Zemack-Rugar, Bettman, \& Fitzsimons, 2007). While 
prior research has focused primarily on indulgent hedonic food products, we expand these findings demonstrating sugar as a desired attribute when individuals consume in response to discrete emotions.

Further, research related to guilt and/or sadness within the food domain has primarily focused on psychological explanations of how these discrete emotions impact indulgence. For example, Garg and Lerner (2013) propose that sadness increases feelings of helplessness that lead to hedonic consumption, while Salerno, Laran, and Janiszewski (2014) propose that sadness leads individuals to mitigate future losses and thus indulge as a mood-management technique. We extend this literature by providing evidence that physiological changes also occur in response to guilt and sadness which impacts sugar preferences for both hedonic and non-hedonic foods. Thus, our findings contribute to a more complete understanding of the mental and physical changes that occur when guilt and sadness become salient.

\subsection{Practical Implications}

Our research also provides important practical implications. Policy makers and health care practitioners can use the results of this research to better educate consumers on the role that specific emotions play in food consumption behaviors. For example, nutritional interventions should focus on better understanding the discrete emotions that an individual is experiencing relative to assessing the overall valence of one's affective state or motivation to maintain a healthy diet. By recognizing and identifying these discrete emotions, nutritional plans can be implemented that control sugar and other potentially harmful nutrients which may reduce the potential development of health diseases such as diabetes and obesity. In addition, recognizing the impact of various emotions can aid in not only educating consumers to make them more 
aware, but correcting poor food related behaviors as well. This type of education could be particularly impactful during the formative teenage years where food consumption behaviors are developed and persuasive intent become recognized. The findings also lend further support to research that has suggested positive mood induction as a tool to influence food consumption behaviors of those with various eating disorders (Cardi, Leppanen, \& Treasure, 2015).

Given the important role that discrete emotions play in impacting food choices, nutritional interventions may also focus on training consumers in understanding and managing their emotions as a tool to make healthier consumption decisions. For example, Kidwell, Hasford, and Hardesty (2015) provide guidelines for training emotional intelligence (EI) and demonstrate how EI training, relative to nutrition knowledge training, can lead to weight loss over a 3-month period. These effects are attributed to a more systematic processing of one's emotions associated with food relative to a more typical reliance on implicit food associations (e.g., unhealthy = tasty; Raghunathan, Naylor, \& Hoyer, 2006). Through EI training, individuals may learn that the mood-enhancing benefits from consuming sugar only provide a short-term benefit and may generate even more intense negative reactions and feelings of helplessness over time. Thus, we advocate that nutritional interventions consider a more comprehensive approach that not only educates individuals about the nutritional content of food, but that also considers the emotions associated with consumption and how those feelings can impact dieters over time.

Additionally, marketing practitioners can use this knowledge of what specific product attributes are sought when developing persuasive appeals associated with food. Our studies demonstrated that guilt will limit consumption of foods perceived as high-sugar indulgences. However, if a product was positioned as an alternative to a high-sugar product, a message emphasizing a lack of guilt upon consumption may be particularly effective. For instance, a 
sugar-free syrup marketed to parents could emphasize that the product will deliver the same great taste as traditional syrup without the guilt of giving one's child an unhealthy food. Numerous traditionally high-sugar food products have introduced line extensions using alternative forms of sweeteners and natural sugars like cane sugar and stevia. Products that have attempted such strategies include Yoplait yogurts, where the original Strawberry Banana 6 oz cup contains 27 grams of sugar and the light version contains 14 grams; and Coca-Cola, where a can of the original soda contains 39 grams of sugar while Coca-Cola Life, which uses cane sugar and stevia contains 24 grams of sugar. Thus, message framing that emphasizes a connection between guilt and low-sugar could be used for a wide variety of products.

Finally, since our findings suggest that sadness will increase the desire for sugar, typical high sugar products such as sweets and chocolates can use sad appeals to position their products as a way for consumers to feel better. For example, a promotion for ice cream may use an appeal where a child is sad after losing a soccer game and the ice cream is a way to feel better while enjoying time with their family. Another use could be a chocolate gift service that depicts someone having a bad day and the gift makes them smile.

\subsection{Limitations and Future Research}

While our findings provide initial insights into how discrete emotions influence specific macronutrient consumption, several areas should continue to be explored. First, our research examines challenge and threat stressors via guilt and sadness. Future research should examine other challenge and threat stressors as well to provide additional support for our conceptual model. For example, attentiveness and anger are related to challenge and threat stressors, respectively (Adam \& Epel, 2007; Rodell \& Judge, 2009). While we would expect similar results 
across these discrete emotions, future research should continue to examine these emotions to provide additional support for our theoretical model.

Research should also examine how discrete emotions that are positive in valence impact macronutrient consumption. For example, positive emotions such as hopefulness and happiness differ in their arousal (Winterich \& Haws, 2011). These differences in positive arousal could produce distinct physiological responses, such as differences in the release of dopamine and other neurochemicals (Kringelbach \& Berridge, 2009; Xu et al., 2004). These physiological changes may also impact preference for carbohydrates in the form of sugar and other macronutrients as well.

Our studies highlight how sugar, relative to guilt, can lead to an increase in carbohydrate consumption. However, studies of food choice have identified that feelings of guilt, particularly when it is associated with consumption, can also lead individuals to consume unhealthy foods and fail in their dietary goals over time (Goldsmith, Cho, \& Dhar, 2012; Kuijer, Boyce, \& Marshall, 2015). Future research should examine what specific macronutrients individuals experiencing guilt tend to consume, and what factors contribute to unhealthy consumption patterns within these individuals. The impact of portion presentation on food consumption has also been linked with negative affect (Bui, Tangari, \& Haws, 2017). Thus, examining the entanglement of emotions, nutrient composition, and food presentation is a fruitful area for extending this research.

Future research could extend our findings by exploring the effects of emotions on the intake of other macronutrients, such as fat and protein. For example, might certain positive and negative emotions motivate an individual to strengthen themselves by consuming greater amounts of protein? Furthermore, is the desire to consume protein linked to physiological 
changes in the same manner as sugar? By examining these factors, a deeper knowledge of the link between discrete emotions and macronutrient intake can be established that provides insights on how and why individuals cope with their affective states through food consumption.

Lastly, individual factors such as demographics and dietary restraint were not captured in the current research. As previous research has found links with food consumption behaviors and individual factors, future research should attempt to expand our findings to specific groups. For example, what demographics may be at a greater risk of overconsuming sugar in response to negative affective states? Given the important role of discrete negative emotions in macronutrient consumption, we hope that future research continues examining the important role that emotions play in the food choices of consumers. 


\section{Appendix}

\section{STUDY STIMULI AND SELECT MEASURES}

Studies 2 and 3

Sentence Unscramble Prime

Guilt

Friends / felt guilty /Matt / for/ deceiving / his

My/ all/ fault / was / it

I blame / guilty / feeling / for / myself

Stopped / I could / have / happened / what

Guilty / what / I said / about I feel

\section{Sadness}

What / felt sad/ Matt / about / happened

Missed / I'm sad / out / I

It's / to think / sad / about it / for me

Feeling / out of / sad / is / control / my

Upset / remember / I get / when I /

\section{Study 2}

Process Evidence Measures/ Sugar Craving Items

"Right now, I would like to eat something high in sugar."

$1=$ Strongly Disagree, 7 = Strongly Agree

"Consuming something sweet would put me in a positive mood."

$1=$ Strongly Disagree, $7=$ Strongly Agree

"I would like a sugary snack when I finish this."

$1=$ Strongly Disagree, $7=$ Strongly Agree

\section{Study 3}

High Sugar Yogurt Label

\section{VANILLA CRÈME YOGURT}

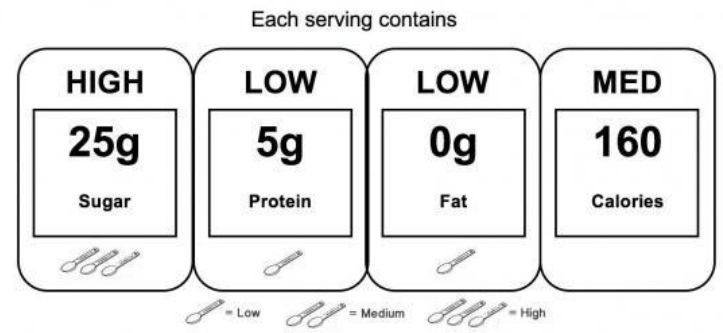


Low Sugar Yogurt Label

\section{VANILLA CRÈME YOGURT}

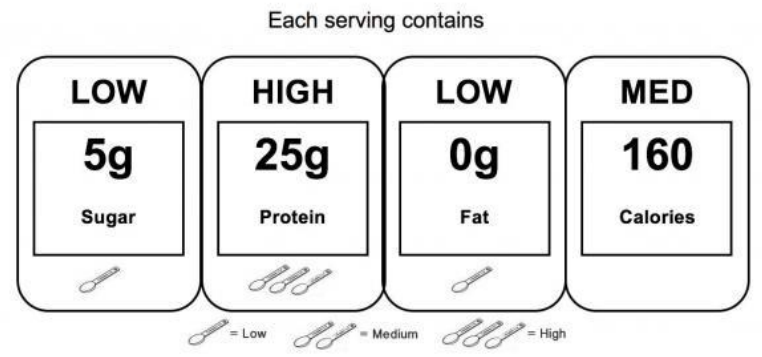

Study 4

Display of Build-Your-Own-Shake Bar

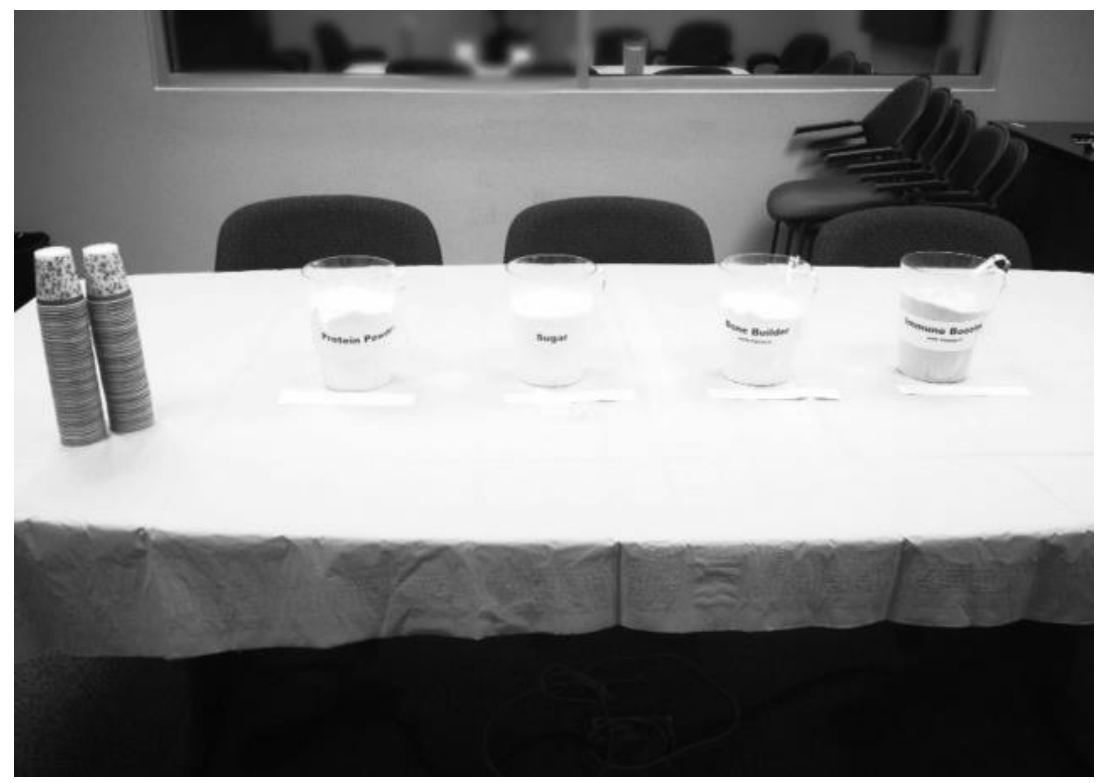




\section{References}

Adam, T. C., \& Epel, E. S. (2007). Stress, eating and the reward system. Physiology \& Behavior, 91(4), 449-458.

Allard, T., \& White, K. (2015). Cross-domain effects of guilt on desire for self-improvement products. Journal of Consumer Research, 42(3), $401-419$.

American Heart Association. (2018). Frequently asked questions about sugar. Retrieved from http://www.heart.org/HEARTORG/HealthyLiving/HealthyEating/HealthyDietGoals/Freq uently-Asked-Questions-About-Sugar_UCM_306725_Article.jsp\#.W5kg1-hKiUk

Aydinoğlu, N. Z., \& Krishna, A. (2011). Guiltless gluttony: The asymmetric effect of size labels on size perceptions and consumption. Journal of Consumer Research, 37(6), 1095-1112.

Baumeister, R. F., Heatherton, T. F., \& Tice, D. M. (1994). Losing Control: How and Why People Fail at Self-Regulation. San Diego: Academic Press.

Blascovich, J. (2013). Challenge and Threat. Handbook of approach and avoidance motivation, $431-444$.

Blascovich, J., Mendes, W. B., Hunter, S. B., Lickel, B., \& Kowai-Bell, N. (2001). Perceiver threat in social interactions with stigmatized others. Journal of Personality and Social Psychology, 80(2), 253-267.

Blascovich, J., \& W. B. Mendes (2000). Challenge and threat appraisals: The role of affective cues. In J. Forgas (Ed.), Feeling and Thinking: The role of affect in social cognition (pp. 59-82). Cambridge UK: Cambridge University Press.

Blascovich, J., \& Tomaka, J. (1996). The biopsychosocial model of arousal regulation. Advances in Experimental Social Psychology, 28(1), 1-51.

Briley, D. A., \& Aaker, J. L. (2006). When does culture matter? Effects of personal knowledge on the correction of culture-based judgments. Journal of Marketing Research, 43(3), 395408.

Bublitz, M. G., Peracchio, L. A., \& Block, L. G. (2010). Why did I eat that? Perspectives on food decision making and dietary restraint. Journal of Consumer Psychology, 20(3), 239-258.

Bui, M. M., Tangari, A. H., \& Haws, K. L. (2017). Can health "halos" extend to food packaging? An investigation into food healthfulness perceptions and serving sizes on consumption decisions. Journal of Business Research, 75, 221-228.

Bunim, J. (2013). Quantity of sugar in food supply linked to diabetes rates. University of California San Francisco. Retrieved from 
https://www.ucsf.edu/news/2013/02/13591/quantity-sugar-food-supply-linked-diabetes$\underline{\text { rates }}$

Cardi, V., Leppanen, J., \& Treasure, J. (2015). The effects of negative and positive mood induction on eating behavior: A meta-analysis of laboratory studies in the healthy population and eating and weight disorders. Neuroscience \& Biobehavioral Reviews, 57, $299-309$.

Carveth, D. L. (2001). The unconscious need for punishment: Expression or evasion of the sense of guilt? Psychoanalytic Studies, 3(1), 9-21.

Centers for Disease Control and Prevention (CDC) (2016). The health effects of overweight and obesity. Center for Disease Control and Prevention. Retrieved from https://www.cdc.gov/healthyweight/effects/index.html

Center for Disease Control and Prevention (CDC). (2017a). New CDC report: More than 100 million Americans have diabetes or prediabetes. Center for Disease Control and Prevention. Retrieved from https://www.cdc.gov/media/releases/2017/p0718-diabetesreport.html

Center for Disease Control and Prevention (CDC). (2017b). Prevalence of obesity among adults and youth: United States, 2015 - 2016. Center for Disease Control and Prevention, Retrieved from https://www.cdc.gov/nchs/data/databriefs/db288.pdf.

Cools, J., Schotte, D. E., \& McNally, R. J. (1992). Emotional arousal and overeating in restrained eaters. Journal of abnormal psychology, 101(2), 348.

Cornil, Y., \& Chandon, P. (2013). From fan to fat? Vicarious losing increases unhealthy eating, but self-affirmation is an effective remedy. Psychological Science, 24(10), 1936-1946.

Dallman, M. F. (2010). Stress-induced obesity and the emotional nervous system. Trends in Endocrinology \& Metabolism, 21(3), 159-165.

Dickerson, S. S., Mycek, P. J., \& Zaldivar, F. (2008). Negative social evaluation, but not mere social pressure, elicits cortisol response to a laboratory stressor task. Health Psychology, $27(1), 116-121$.

Dunn, L., \& Hoegg, J. (2014). The impact of fear on emotional brand attachment. Journal of Consumer Research, 41(1), $152-168$.

Epel, E., Lapidus, R., McEwen, B., \& Brownell, K. (2001). Stress may add bite to appetite in women: a laboratory study of stress-induced cortisol and eating behavior. Psychoneuroendocrinology, 26(1), 37-49.

Ershadi, M., Russell, J. A., Herz, R. S. (2017). The (non)-effect of induced emotion on desire for different types of foods. Food Quality and Preference, 62, 214- 217. 
Gaab, J., Rohleder, N., Nater, U. M., \& Ehlert, U. (2005). Psychological determinants of the cortisol stress response: the role of anticipatory cognitive appraisal. Psychoneuroendocrinology, 30(6), 599-610.

Gardner, M., Wansink, B., Kim, J., \& Park, S. B. (2014). Better moods for better eating; How mood influences food choice. Journal of Consumer Psychology, 24(3), 320-335.

Garg, N., \& Lerner, J. S. (2013). Sadness and consumption. Journal of Consumer Psychology, 23(1), 106-113.

Garg, N., Wansink, B., \& Inman, J. J. (2007). The influence of incidental affect on consumers' food intake. Journal of Marketing, 71(1), 194-206.

Giner-Sorolla, R. (2001). Guilty pleasures and grim necessities: Affective attitudes in dilemmas of self-control. Journal of Personality and Social Psychology, 80(2), 206.

Goldberg, J. H., Lerner, J. S., \& Tetlock, P. E. (1999). Rage and reason: The psychology of the intuitive prosecutor. European Journal of Social Psychology, 29(56), 781-795.

Goldsmith, K., Cho, E. K., \& Dhar, R. (2012). When guilt begets pleasure: the positive effect of a negative emotion. Journal of Marketing Research, 49(6), 872-881.

Han, D., Duhachek, A., \& Agrawal, N. (2014). Emotions shape decisions through construal level: The case of guilt and shame. Journal of Consumer Research, 41(4), 1047-1064.

Harvard School of Public Health. (2012). Fact sheet: Sugary drink supersizing and the obesity epidemic. Retrieved from https://cdn1.sph.harvard.edu/wpcontent/uploads/sites/30/2012/10/sugary-drinks-and-obesity-fact-sheet-june-2012-thenutrition-source.pdf

Harvey, A., Nathens, A. B., Bandiera, G., \& LeBlanc, V. R. (2010). Threat and challenge: cognitive appraisal and stress responses in simulated trauma resuscitations. Medical Education, 44(6), 587-594.

Hayes, A.F. (2013). Introduction to mediation, moderation, and conditional process analysis: A regression-based approach. Guilford Press.

Hung, I. W., \& Mukhopadhyay, A. (2012). Lenses of the heart: How actors' and observers' perspectives influence emotional experiences. Journal of Consumer Research, 38(6), 1103-1115.

Kidwell, B., Hasford, J., \& Hardesty, D. M. (2015). Emotional ability training and mindful eating. Journal of Marketing Research, 52(1), 105-119. 
King, G. A., Herman, C. P., \& Polivy, J. (1987). Food perception in dieters and non-dieters. Appetite, 8(2), 147-158.

Kringelbach, M. L., \& Berridge, K. C. (2009). Towards a functional neuroanatomy of pleasure and happiness. Trends in Cognitive Sciences, 13(11), 479-487.

Kouchaki, M., Oveis, C., \& Gino, F. (2014). Guilt enhances the sense of control and drives risky judgments. Journal of Experimental Psychology: General, 143(6), 2103.

Kuijer, R. G., \& Boyce, J. A. (2014). Chocolate cake. Guilt or celebration? Associations with healthy eating attitudes, perceived behavioural control, intentions and weight-loss.

Appetite, 74, 48-54.

Kuijer, R. G., Boyce, J. A., \& Marshall, E. M. (2015). Associating a prototypical forbidden food item with guilt or celebration: Relationships with indicators of (un) healthy eating and the moderating role of stress and depressive symptoms. Psychology \& Health, 30(2), 203217.

Lazarus, R. S. (1991). Emotion and adaptation. Oxford University Press on Demand.

Lazarus, R. S., \& Folkman, S. (1984). Coping and adaptation. The Handbook of Behavioral Medicine, 282-325.

Leith, K. P., \& Baumeister, R. F. (1996). Why do bad moods increase self-defeating behavior? Emotion, risk tasking, and self-regulation. Journal of Personality and Social Psychology, 71(6), 1250-1267.

Lerner, J. S., Li, Y., \& Weber, E. U. (2012). The financial costs of sadness. Psychological Science, 24(1), 72-79.

Lowe, M. R., \& Fisher Jr, E. B. (1983). Emotional reactivity, emotional eating, and obesity: A naturalistic study. Journal of Behavioral Medicine, 6(2), 135-149.

Macht, M. (1999). Characteristics of eating in anger, fear, sadness, and joy. Appetite, 33, 129 139.

Mantau, A., Hattula, S., \& Bornemann, T. (2018). Individual determinants of emotional eating: A simultaneous investigation. Appetite, 130, 93 - 103.

Nederkoorn, C., Smulders, F. T. Y., \& Jansen, A. (2000). Cephalic phase responses, craving and food intake in normal subjects. Appetite, 35(1), 45-55.

O’Connor, D. B., Jones, F., Connor, M., McMillan, B., \& Ferguson, E. (2008). Effects of daily hassles and eating style on eating behavior. Health Psychology, 27(1), S20 - S31. 
Pieters, R. (2013). Bidirectional dynamics of materialism loneliness: Not just a vicious cycle. Journal of Consumer Research, 40(4), 615 - 631.

Raghunathan, R., Naylor, R. W., \& Hoyer, W. D. (2006). The unhealthy= tasty intuition and its effects on taste inferences, enjoyment, and choice of food products. Journal of Marketing, 70(4), 170-184.

Reichenberger, J. et al. (2018). No haste, more taste: An EMA study of the effects of stress, negative and positive emotions on eating behavior. Biological Psychology, 131, $54-62$.

Rodell, J. B., \& Judge, T. A. (2009). Can "good" stressors spark "bad" behaviors? The mediating role of emotions in links of challenge and hindrance stressors with citizenship and counterproductive behaviors. Journal of Applied Psychology, 94(6), 1438-1451.

Roseman, I. J., Wiest, C., \& Swartz, T. S. (1994). Phenomenology, behaviors, and goals differentiate discrete emotions. Journal of Personality and Social Psychology, 67(2), 206-221.

Russek, M., Lora-Vilchis, M. C., \& Islas-Chaires, M. (1980). Food intake inhibition elicited by intraportal glucose and adrenaline in dogs on a 22 hour-fasting/2 hour feeding schedule. Physiology \& behavior, 24(1), 157-161.

Russek, M., Mogenson, G. J., \& Stevenson, J. A. F. (1967). Calorigenic, hyperglycemic and anorexigenic effects of adrenaline and noradrenaline. Physiology \& Behavior, 2(4), 429433.

Russek, M., Stevenson, J. A. F., \& Mogenson, G. J. (1968). Anorexigenic effects of adrenaline, amphetamine, and FMS 1A. Canadian journal of physiology and pharmacology, 46(4), 635-638.

Salerno, A., Laran, J., \& Janiszewski, C. (2014). Hedonic eating goals and emotion: When sadness decreases the desire to indulge. Journal of Consumer Research, 41(1), 135 - 151.

Smith, C. A., \& Ellsworth, P. C. (1985). Patterns of cognitive appraisal in emotion. Journal of Personality and Social Psychology, 48(4), 813-838.

Srull, T. K., \& Wyer, R. S. (1979). The role of category accessibility in the interpretation of information about persons: Some determinants and implications. Journal of Personality and Social Psychology, 37(10), 1660-1672.

Tangney, J. P., \& Dearing, R. L. (2003). Shame and guilt. Guilford Press.

Tangney, J. P., Miller, R. S., Flicker, L., \& Barlow, D. H. (1996). Are shame, guilt, and embarrassment distinct emotions?. Journal of Personality and Social Psychology, 70(6), 1256. 
Tempel, D. L., \& Leibowitz, S. F. (1994). Adrenal steroid receptors: interactions with brain neuropeptide systems in relation to nutrient intake and metabolism. Journal of Neuroendocrinology, 6(5), 479-501.

Tice, D. M., Bratslavsky, E., \& Baumeister, R. F. (2001). Emotional distress regulation takes precedence over impulse control: If you feel bad, do it!. Journal of Personality and Social Psychology, 80(1), 53-67.

Troisi, J. D., \& Gabriel, S. (2011). Chicken soup really is good for the soul "comfort food" fulfills the need to belong. Psychological Science, 22(6), 747-753.

Van Dam, R. M., \& Seidell, J. C. (2007). Carbohydrate intake and obesity. European Journal of Clinical Nutrition, 61, S75-S99.

Watson, L., \& Spence, M. T. (2007). Causes and consequences of emotions on consumer behaviour: A review and integrative cognitive appraisal theory. European Journal of Marketing, 41(5), 487-511.

Winterich, K. P., \& Haws, K. L. (2011). Helpful hopefulness: The effect of future positive emotions on consumption. Journal of Consumer Research, 38(3), 505-524.

Xu, Y.L., Reinscheid, R.K., Huitron-Resendiz, S., Clark, S.D., Wang, Z., Lin, S.H., Brucher, F.A., Zeng, J., Ly, N.K., Henriksen, S.J. and de Lecea, L. (2004). Neuropeptide S: a neuropeptide promoting arousal and anxiolytic-like effects. Neuron, 43(4), pp.487-497.

Yanovski, S. (2003). Sugar and fat: cravings and aversions. The Journal of Nutrition, 133(3), 835-837.

Zemack-Rugar, Y., Bettman, J. R., \& Fitzsimons, G. J. (2007). The effects of nonconsciously priming emotion concepts on behavior. Journal of Personality and Social Psychology, 93(6), 927. 
Figure 1. Effect of Discrete Emotion on Sugar Preference through Sugar Cravings

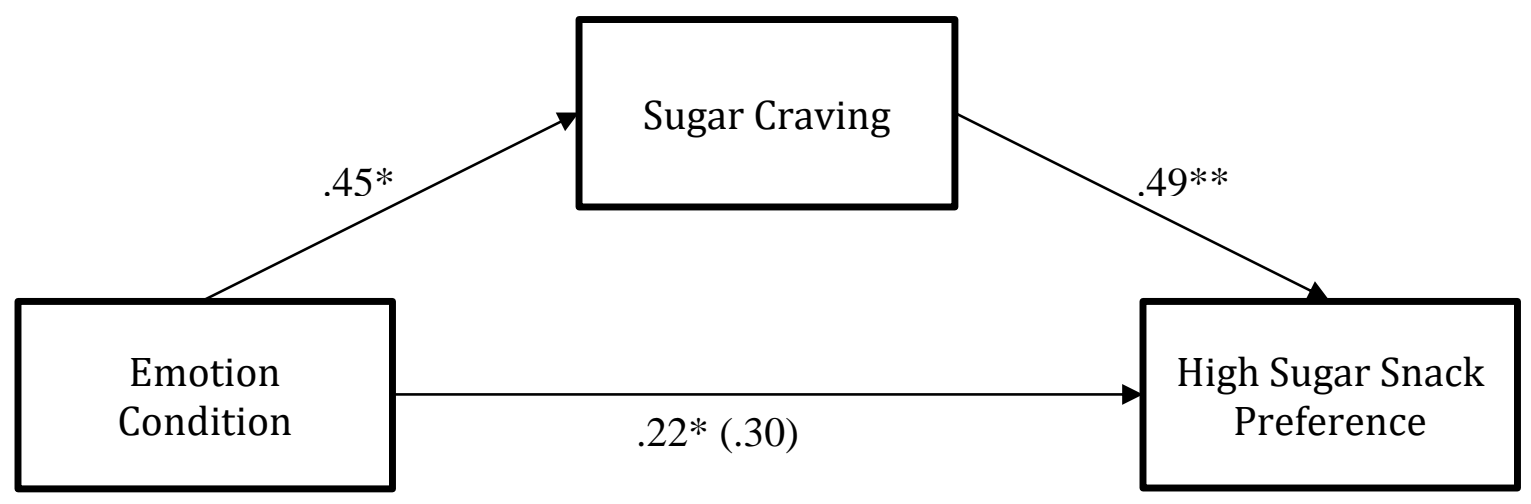

$* p<.05, * * p<.001$

Figure 2. Preference for High versus Low Sugar Snack by Discrete Emotion

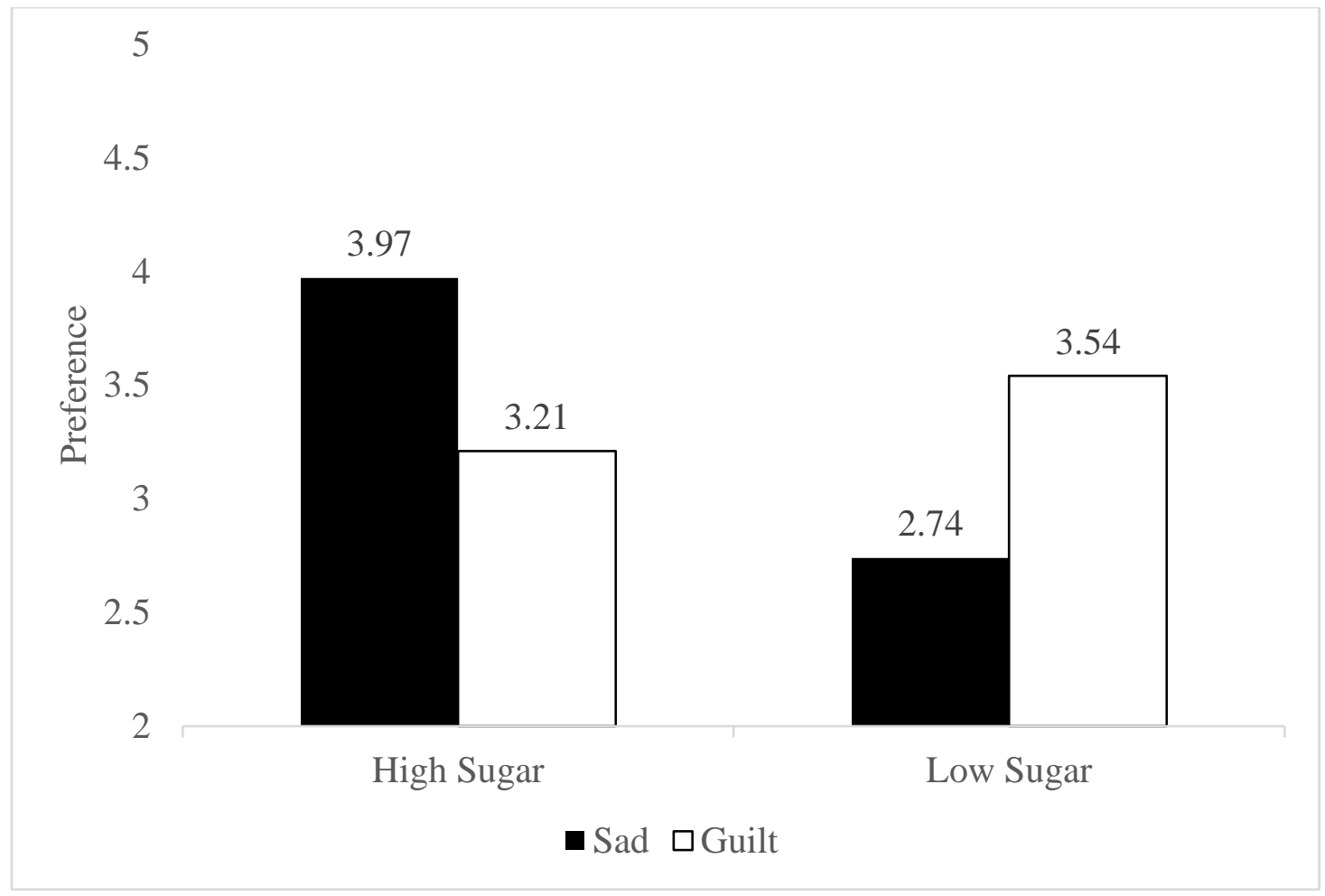

